International Journal of Engineering \& Technology, $7(4.11)(2018) 40-43$
International Journal of Engineering \& Technology
SPC
Website: www.sciencepubco.com/index.php/IJET
Research paper

\title{
Investigation of Human Electromagnetic Radiation Characteristic For Kidney Disease Patients
}

\author{
Siti Zura A. Jalil ${ }^{1}$, Aimi Nazerah Shamsuddin ${ }^{1}$, Siti Armiza Mohd Aris ${ }^{1}$, Nurul Aini Bani ${ }^{1}$, Hazilah Mad Kaidi ${ }^{1}$, \\ Mohd Nabil Muhtazaruddin ${ }^{1}$, Sahnius Usman ${ }^{1}$, Mohd Azri Mohd Izhar ${ }^{1}$, Rihana Yusuf ${ }^{2}$, Ros Syilawani S. Abdul \\ Kadir $^{2}$ \\ ${ }^{1}$ Razak Faculty of Technology and Informatics, Universiti Teknologi Malaysia, Kuala Lumpur, Malaysia \\ ${ }^{2}$ Faculty of Electrical Engineering, Universiti Teknologi MARA, Shah Alam, Selangor, Malaysia \\ *Corresponding author E-mail: sitizura.kl@utm.my
}

\begin{abstract}
The human body is shown to have their own radiation that emits at certain frequencies into the space surround the body. The purpose of this paper is to investigate the characteristic of human electromagnetic radiation among kidney disease patients and non-kidney disease participants. The body radiation frequencies are measured using body radiation wave detector at twenty-two points of the human body. The properties of human electromagnetic radiation are evaluated using statistical analysis of dependent t-test of Wilcoxon Signed Rank test and independent t-test of Mann-Whitney U test. Significant results with the Sig. value below 0.05 are shown in lower body, torso, chakra, arm and upper body, thus indicates the characteristic differences of human electromagnetic radiation frequency between kidney disease patients and non-kidney disease participants.
\end{abstract}

Keywords: Human electromagnetic radiation; frequency; kidney disease; $t$-test.

\section{Introduction}

\subsection{Kidney Disease}

In human anatomy, kidneys are found on either side of the spine below the ribs and it function to clean the blood by acting as a filter to remove waste and excess fluid from the blood, electrolyte balance and also involved in regulating blood pressure in the body. The kidney disease would happen when the kidneys does not work properly, especially for those who have higher risk factors that having diabetes and/or hypertension. If the kidneys damage, waste and fluid will build-up in the body causing swelling in the ankles, weakness, poor sleep, vomiting and shortness of breath. Kidney disease, also known as renal failure is described as the gradual loss of kidney function. Chronic kidney disease (CKD) is commonly referred to a loss of kidney function for at least three months and poses a major health problem. Initially, kidney failure may cause no symptoms. Late detection of kidney failure can cause kidney damage and loss of kidney function. Common detection technique of kidney disease is measured directly via blood test on glomerular filtration rate and indirectly via urine and electrolyte levels. However, those techniques are invasive, tedious, and complex [1]. Besides, the tests is not recommended for the general population and is performed to the patient with higher risk factors that having diabetes and/or hypertension [2]. Since the patient often present late with complications of CKD, early identification, treatment and prevention of chronic kidney disease will be necessary to reduce the burden of the disease.

\subsection{Human Electromagnetic Radiation}

The electromagnetic radiation of the human has shown to represents a unique feature of the human body. The electromagnetic radiation produced by the human body is associated with electrical systems of the body $[3,4]$ and could be due to the existence of the nervous system in the body as it is made up of a network of nerve cells or neurons that communicate with each other and with other cells in the body. Of course, the communication process is complex and done in a very fast speed through the electromagnetic waves and photons in the cells, between cells, between relevant bodies, and also from the communications via nerve fibers, hormones and other molecules [5]. As a biological system, the human electromagnetic radiation is varying in accordance with health and activity of the body $[6,7]$. Furthermore, previous studies have shown human body has a different characteristic of electromagnetic radiation between gender [8,9] and significant results also have been found for stroke and non-stroke participants using electromagnetic radiation from human [10].

In this study, a new technique that could be used to identify kidney failure by using electromagnetic radiation of the human body is proposed. For the purpose of measurement, frequency detector is used to measure human electromagnetic radiation from kidney disease patients and non-kidney disease participants. This technique has successfully been used for detection of electromagnetic radiation of living bodies in several studies [10-12].

For analysis, t-test is employed to identify the characteristic of human electromagnetic radiation between kidney disease patients and non-kidney disease participants. The t-test is a statistical test that able to compare groups and commonly used in identifying features in several studies [13, 14]. The outcome of this study is 
expected to compare the characteristic of human electromagnetic radiation frequency of kidney failure patients and non-kidney disease participant. Besides, the study is also expected to be used in the future as an alternative technique of non-invasive and painless to identify kidney failure and will facilitating the diagnosis procedure.

\section{Methodology}

The methodology for this paper is comprised of three parts. The first part is data acquisition of human electromagnetic radiation frequency on kidney-disease patients and non-kidney disease participants. Based on mean of frequencies data, the second part is preliminary analysis of descriptive statistical test to assess the data distributions. Then, the third part is exploring differences between means using statistical t-test to identify the characteristics of human electromagnetic radiation.

\subsection{Data Collection}

The human electromagnetic radiation frequency is examined on twenty-two points surround the human body as shown in Fig. 1. Fourty-one participants were involved in this study, which consists of thirty-one kidney-disease patients and ten non-kidney disease participants. For kidney disease patients, the human radiation frequencies were taken two times on before and after the hemodialysis. The frequency of human electromagnetic radiation is measured using a hand held frequency meter namely body radiation wave detector. The detector is able to detect a wide range of human electromagnetic radiation frequencies in range of Mega Hertz. During the measurement, the length of the antenna for the detector was set at the $7^{\text {th }}$ segment and is placed at a distance of 1 $\mathrm{cm}$ to $5 \mathrm{~cm}$ above the body for each point. The frequency for each particular point on each participant is collected three times in order to ensure it reliability. Besides, all frequency data of patients and participants are obtained at the same location in the dialysis center and the ambient frequencies are also monitored which it measured immediately before and after the experiment. The detected frequency data is then transferred to a computer for storage and offline data analysis. The illustration example for measurement technique is shown in Fig. 2.

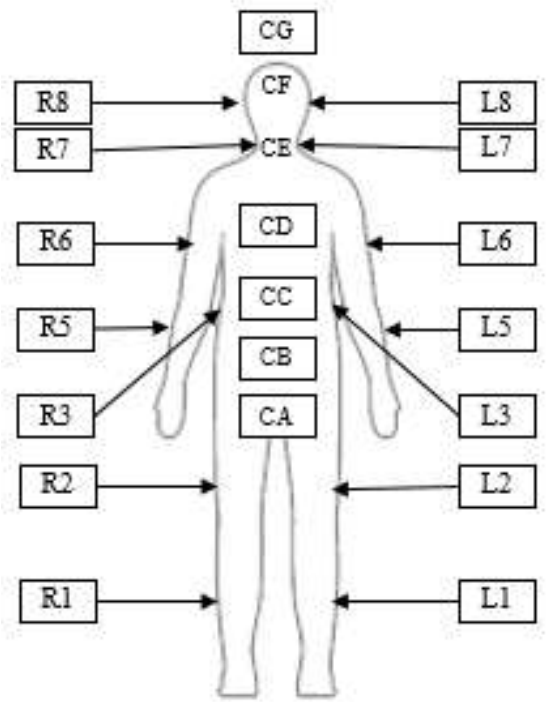

Fig. 1: Points of human body radiation for data collection

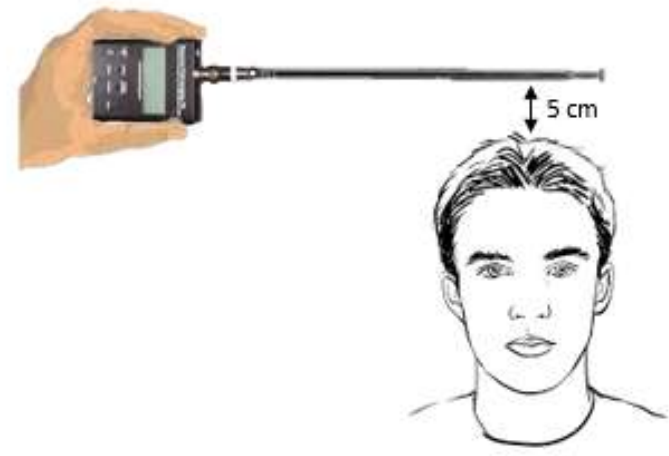

Fig. 2: The measurement technique

\subsection{Preliminary Analysis}

In statistical analysis, most of the outcomes are underlying the assumption of normal distribution. The descriptive statistical test is conducted to assess the distribution of data and examine the data normality. Normality is symmetrical pattern of data distribution for an individual continuous variable and corresponds to the bell-shaped curve. In this preliminary analysis, the tests of normality are assessed using Kolmogorov-Smirov statistic with alpha level of .05. A non-significant result for any value exceeds 0.05 indicates normality.

\subsection{Data Analysis}

The characteristic differences of human electromagnetic radiation frequency of kidney disease patients and non-kidney disease participants are explored using the statistical analysis of t-test. The ttest is employed to compare mean of two groups and there are two types of t-test that is dependent and independent t-tests. Dependent or paired t-test is used to examine the characteristic differences between kidney disease participants on before and after the hemodialysis. Meanwhile, the independent t-test is applied to distinguish the characteristic between kidney disease patients and nonkidney disease participants. As the parametric statistical paired and independent t-tests having stringent assumption on data normality and minimum number of samples, alternatively, a nonparametric method of t-test is employed in this study that is Wilcoxon Signed Rank test and Mann-Whitney U test. A Wilcoxon Signed Rank test and Mann-Whitney U test are useful techniques to compare means for small samples, able to tolerate if the data distribution is skewed and may produce result exact as parametric test. The assumptions for these tests are random samples and independent observations.

Besides, due to a limited number of participants for non-kidney disease, a synthetic data is generated randomly by modifying the original dataset of non-kidney disease participants for not more than $10 \%$ and then applied in the analysis. The number of nonkidney disease participants is limited due to less number of nonkidney disease people visiting the dialysis center as all the measurements are performed in the dialysis center to produce similar background and to ensure the data reliability as well. Synthetic data is used to improve the number of samples in order to compare the mean efficiently [15]. Synthetic data has been effectively used in other studies when the data is limited [16] and able to achieve good results [17].

\section{Results and Discussion}

The frequency data acquired from each participant is obtained under controlled environment and all participants have similar comfort level to ensure consistency and accuracy of reading frequency. The ambient frequencies are also monitored, which it measured immediately before and after the experiment. 
In preliminary data analysis, the tests of normality are assessed using Kolmogorov-Smirov statistic. The inspection of normality tests is analyzed separately for each variable between disease and non-disease participants in order to examine their individual distribution. Table 1 (a)-(b) show the result for normality tests on some of the variables for kidney disease patients and non-kidney disease participants respectively. As an alpha level of 0.05 is used in the analysis, a non-normal distribution is shown in most of the variables, thus violate the assumption of normality.

Table 1: Kolmogorov-Smirov Tests of Normality

\begin{tabular}{|c|c|c|c|c|c|c|c|}
\hline \multicolumn{4}{|c|}{ (a) Kidney disease patients } & \multicolumn{4}{|c|}{ (b) Non disease participants } \\
\hline & Statistic & $\mathrm{df}$ & Sig. & & Statistic & $\overline{\mathrm{df}}$ & Sig. \\
\hline $\mathrm{CA}$ & .180 & 31 & .012 & $\mathrm{CA}$ & .177 & 30 & .018 \\
\hline $\begin{array}{c}\text { CC- } \\
\text { B }\end{array}$ & 192 & 31 & .005 & $\begin{array}{c}\text { CC- } \\
\text { B }\end{array}$ & .175 & 30 & 019 \\
\hline $\mathrm{CD}$ & .142 & 31 & .114 & $\mathrm{CD}$ & 213 & 30 & .001 \\
\hline R1 & .225 & 31 & $<.001$ & R1 & .171 & 30 & .026 \\
\hline $\mathrm{R} 2$ & .293 & 31 & $<.001$ & R2 & .146 & 30 & .104 \\
\hline L7 & .337 & 31 & $<.001$ & L7 & .236 & 30 & $<.001$ \\
\hline L8 & .215 & 31 & .001 & L8 & .143 & 30 & .118 \\
\hline
\end{tabular}

Therefore, Wilcoxon Signed Rank test and Mann-Whitney U test techniques are used for further analysis to compare means. Wilcoxon Signed Rank test is used to examine the characteristic differences between kidney disease patients on before and after the hemodialysis, while Mann-Whitney $U$ test is used to distinguish the characteristic between kidney disease patients and non-kidney disease participants. In these tests, criterion of alpha level 0.05 is used as a cut-off point.

Table 2 shows a results of Wilcoxon Signed Rank test. The null hypothesis of this experiment is the median of differences between variable before and after are equals to zero. Significant results that reject null hypothesis with the Sig. value below 0.05 are found in some of the points (Table 2). Statistically significant different are shown in torso and chakra (CA, CC-B, CD, R3), arm (L5), lower body (R1, R2) and upper body (L7, R7, R8, CG).

Table 2: Wilcoxon Signed Rank test

\begin{tabular}{|c|c|}
\hline Human Body Point & Sig. \\
\hline CA & $<.001$ \\
\hline CC-B & $<.001$ \\
\hline CD & .0015 \\
\hline R3 & $<.001$ \\
\hline L5 & .002 \\
\hline R1 & .004 \\
\hline R2 & $<.001$ \\
\hline L7 & .0034 \\
\hline R7 & $<.001$ \\
\hline R8 & .019 \\
\hline CG & .016 \\
\hline
\end{tabular}

In comparing the characteristic between kidney disease patients and non-kidney disease participants, a statistical of Mann-Whitney $\mathrm{U}$ test is then employed. Table 3 shows the results for kidney disease patients (before hemodialysis) and non-kidney disease participants, while Table 4 shows the results for kidney disease patients (after hemodialysis) and non-kidney disease participants. In Table 3 , significant results with the Sig. value below 0.05 are shown in Chakra (CC-B and CF), lower body (L1) and upper body (R8), indicating the mean distribution in these point are different between kidney disease patients and non-kidney disease participant. However, in Table 4, significant results are presented in chakra (CA and CF), arm (L5) and torso (R3).

Table 3: Mann-Whitney U test (before hemodialysis)

\begin{tabular}{|c|c|}
\hline Human Body Point & Sig. \\
\hline CC-B & $<.001$ \\
\hline L1 & .032 \\
\hline R8 & .004 \\
\hline CF & .027 \\
\hline
\end{tabular}

Table 4: Mann-Whitney U test (after hemodialysis)

\begin{tabular}{|c|c|}
\hline Human Body Point & Sig. \\
\hline CA & .023 \\
\hline R3 & $<.001$ \\
\hline L5 & .033 \\
\hline CF & .001 \\
\hline
\end{tabular}

As mention earlier, the variations of reading frequency obtained around the human body related to the changes in physiological activities of the human body. As most of the kidney disease patients suffered with leg pain, abdominal pain, low back pain, chest pain and headache [18], significant results also have been shown in this study on lower body, torso, chakra, arm and upper body. Leg pain such as swelling and cramp are commonly occurred in kidney disease patients and this is in line with the finding that human electromagnetic radiation in the lower body part, i.e. the leg, labelled as L1, R1 and R2 are observed differ between nondisease participants and disease patients before dialysis and also differ between before and after dialysis for kidney patients, respectively. For low back pain and chest pain, the characteristics of human electromagnetic radiation frequency has been distinguished in CC-B, R3 and CD that represent as lower back, waist and heart position in the physical body. Besides, previous study also shown relationship between chronic kidney disease and cardiovascular abnormalities [19]. In arm, significant difference of human electromagnetic radiation frequency has been shown in left arm, L5. For the hemodialysis patients, there is vascular access that is used to remove and return blood during hemodialysis name as arteriovenous (AV) fistula which is located surgically at the arm of the patient (mostly at the left arm). For headache, the characteristic differences of human electromagnetic radiation frequency have been shown in upper body part on R8, CF and CG.

In overall, the initial finding from this experiment shows promising results that will contribute to the body of knowledge in human electromagnetic radiation concept. Of course, many needs to be explore and still far from complete. Further study is to investigate for the other points that are significant and acquire more data to perform further analysis.

\section{Conclusion}

This study investigates the characteristic of human electromagnetic radiation frequency among kidney disease patients and nonkidney disease participants that measured using body radiation wave detector on twenty-two points of the human body. Statistical analysis of Wilcoxon Signed Rank test and Mann-Whitney U test is used to compare electromagnetic radiation on kidney disease patients and non-kidney disease participants. Significant results with the Sig. value below 0.05 are shown in lower body, torso, chakra, arm and upper body. This finding indicates the characteristic differences of human electromagnetic radiation frequency between kidney disease patients and non-kidney disease participants. Further investigations with more data will be performed to distinguish the human electromagnetic radiation frequency. The finding from this study is expected to be used in the future as an alternative technique of non-invasive and painless to identify kidney failure and will facilitate the diagnosis procedure.

\section{Acknowledgement}

The authors wish to express their appreciation to Universiti Teknologi Malaysia for supporting this research. This work is supported by Research University Grant Scheme (Grant No. PY/2016/01579) and Ministry of Education (Vot No. 15H51). Next, the highest gratitude to Darul Naim Dialysis Centre for collaboration in this research. 


\section{References}

[1] M. Sherwood and P. A. McCullough, "Chronic kidney disease from screening, detection, and awareness, to prevention," The Lancet Global Health, 4, 288-289, 2016.

[2] J. A. Vassalotti, R. Centor, B. J. Turner, R. C. Greer, M. Choi, and T. D. Sequist, "Practical approach to detection and management of chronic kidney disease for the primary care clinician," The American Journal of Medicine, 129, 153-162, 2016.

[3] D. B. Geselowitz and M. N. Geselowitz, "The bioelectrical century: Bioelectrical engineering and the "inside story" of the electrical century," Proceedings of the IEEE, 87, 1842-1846, 1999.

[4] N. Czegledy, "Bioelectromagnetism: Discrete interpretations," Technoetic Arts: A Journal of Speculative Research, 1, 135-141, 2003.

[5] C. Zhang, "Electromagnetic body versus chemical body," Network, 81, 7-10, 2003.

[6] H. C. Lee, P. W. Khong, and D. N. Ghista, "Bioenergy based medical diagnostic application based on gas discharge visualization," Proceedings of the 27th Annual Conf. Eng. Medicine and Biology, 2005, pp. 1533-1536.

[7] K. G. Korotkov, "Analysis and monitoring of the human energy state with gas discharge visualization technique," Proceedings of the 18th IEEE Symp. Computer-Based Medical Systems, 2005, pp. 431-436.

[8] S. Z. A. Jalil, H. Abdullah, and M. N. Taib, "Human body radiation wave analysis on the human torso," Proceedings of the International Conference on BioSignal Analysis, Processing and Systems, 2015, pp. 22-27.

[9] S. Z. A. Jalil, M. N. Taib, H. A. Idris, and M. M. Yunus, "Classification of human radiation wave on the Upper body segment," Proceedings of the IEEE 3rd International Conference on System Engineering and Technology, 2013, pp. 73-77.

[10] R. S. S. A. Kadir, Z. H. Murat, M. N. Taib, and S. Z. A. Jalil "Investigation of electromagnetics radiation for stroke patients and non-stroke participants," Proceedings of the IEEE 6th Control and System Graduate Research Colloquium, 2015, pp. 130-134.

[11] S. Z. A. Jalil, H. Abdullah, and M. N. Taib, "Detection of endogenous electromagnetic field of the human body," ARPN Journal of Engineering and Applied Sciences, 10, 9650-9658, 2015.

[12] V. Shalatonin, "A study of the endogenous electromagnetic field into the space around the flower plants," Proceedings of the Joint 32nd Int. Conf. Infrared and Millimeter Waves and 15th Int. Conf. Terahertz Electronics, 2007, pp. 293-294.

[13] G. Q. Zhu and P. H. Yang, "Identifying the candidate genes for Alzheimer's disease based on the rejection region of $\mathrm{T}$ test," Proceedings of the International Conference on Machine Learning and Cybernetics, 2016, pp. 732-736.

[14] A. Y. Mahmoudi and A. O. Nejad, "Evaluating the level of awareness of teachers and educational head of principles and methods of content analysis of second-grade high school textbooks," Journal of Fundamental and Applied Sciences, 8(3S), 2172-2180, 2016.

[15] D.-C. Li, S. C. Hu, L.-S. Lin, and C.-W. Yeh, "Detecting representative data and generating synthetic samples to improve learning accuracy with imbalanced data sets," Plos One, 12, 1-24, 2017.

[16] T. Varga and H. Bunke, "Generation of synthetic training data for an HMM-based handwriting recognition system," in Proc. Seventh Int. Conf. Document Analysis and Recognition, 2003, pp. 618-622.

[17] M. Barnard, M. Matilainen, and J. Heikkila, "Body part segmentation of noisy human silhouette images," Proceedings of the IEEE Int. Conf. Multimedia and Expo, 2008, pp. 1189-1192.

[18] Z. H. Bajwa, K. A. Sial, A. B. Malik, and T. I. Steinman, "Pain patterns in patients with polycystic kidney disease," Kidney International, 66, 1561-1569, 2004

[19] R. T. Gansevoort, R. Correa-Rotter, B. R. Hemmelgarn, T. H. Jafar, H. J. L. Heerspink, J. F. Mann, et al., "Chronic kidney disease and cardiovascular risk: Epidemiology, mechanisms, and prevention," The Lancet, 382, 339-352, 2013. 